\title{
Functional Magnetic Resonance Imaging Blood Oxygenation Level-Dependent Signal and Magnetoencephalography Evoked Responses Yield Different Neural Functionality in Reading
}

\author{
Johanna Vartiainen, Mia Liljeström, Miika Koskinen, Hanna Renvall, and Riitta Salmelin \\ Brain Research Unit, MEG Core and Advanced Magnetic Imaging Center, Low Temperature Laboratory, School of Science and Technology, Aalto \\ University, FI-00076 Aalto, Finland
}

It is often implicitly assumed that the neural activation patterns revealed by hemodynamic methods, such as functional magnetic resonance imaging (fMRI), and electrophysiological methods, such as magnetoencephalography (MEG) and electroencephalography (EEG), are comparable. In early sensory processing that seems to be the case, but the assumption may not be correct in high-level cognitive tasks. For example, MEG and fMRI literature of single-word reading suggests differences in cortical activation, but direct comparisons are lacking. Here, while the same human participants performed the same reading task, analysis of MEG evoked responses and fMRI blood oxygenation level-dependent (BOLD) signals revealed marked functional and spatial differences in several cortical areas outside the visual cortex. Divergent patterns of activation were observed in the frontal and temporal cortex, in accordance with previous separate MEG and fMRI studies of reading. Furthermore, opposite stimulus effects in the MEG and fMRI measures were detected in the left occipitotemporal cortex: MEG evoked responses were stronger to letter than symbol strings, whereas the fMRI BOLD signal was stronger to symbol than letter strings. The EEG recorded simultaneously during MEG and fMRI did not indicate neurophysiological differences that could explain the observed functional discrepancies between the MEG and fMRI results. Acknowledgment of the complementary nature of hemodynamic and electrophysiological measures, as reported here in a cognitive task using evoked response analysis in MEG and BOLD signal analysis in fMRI, represents an essential step toward an informed use of multimodal imaging that reaches beyond mere combination of location and timing of neural activation.

\section{Introduction}

Electromagnetic and hemodynamic imaging methods probe neural activity via different measurable quantities. Whereas the functional magnetic resonance imaging (fMRI) blood oxygenation leveldependent (BOLD) signal can yield (sub)millimeter spatial resolution, tracking of neural current with magnetoencephalography (MEG) via the magnetic field provides millisecond-level time information with reasonable spatial resolution. An initial approach in combining extracranial electrophysiological and hemodynamic measures was to assume that both reflect the same neural activity and, thus, by taking the location from fMRI and timing from MEG [or electroencephalography (EEG)], one would procure a more

\footnotetext{
Received May 24, 2010; revised Nov. 5, 2010; accepted Nov. 9, 2010.

This study was financially supported by the Academy of Finland (National Centers of Excellence Programme 2006-2011, personal grants to H.R. and R.S.), the Finnish Ministry of Education (Finnish Graduate School of Neuroscience), the Sigrid Jusélius Foundation, and the European Research Council (Advanced Grant to R. Hari, director of the Brain Research Unit). We thank A. Jalava for providing the script for the ECD surface projections, M. Kujala for advice on the fMRI analysis, and H. Roikola, M. IIIman, M. Kattelus, and J. Kainulainen for help in the measurements and data analysis.

Correspondence should be addressed to Johanna Vartiainen, Brain Research Unit, MEG Core and Advanced Magnetic Imaging Center, Low Temperature Laboratory, School of Science and Technology, Aalto University, P.0. Box 15100, FI-00076 Aalto, Finland. E-mail: juusvuor@neuro.hut.fi.

DOI:10.1523/JNEUROSCI.3113-10.2011

Copyright $\odot 2011$ the authors $\quad 0270-6474 / 11 / 311048-11 \$ 15.00 / 0$
}

complete spatiotemporal picture of neural processing (Dale et al., 2000). However, it is possible that MEG and fMRI are differentially sensitive to various characteristics of neural activity, such as synchronicity or duration of neural activation, or feedforward versus feedback influences on neural responses (Nunez and Silberstein, 2000). In direct comparisons, MEG evoked responses and fMRI BOLD signals have shown fairly good spatial convergence in low-level sensory and motor processing (e.g., Korvenoja et al., 1999; Sharon et al., 2007). Fewer studies have focused on complex cognitive tasks (e.g., Croizé et al., 2004; Liljeström et al., 2009), although any divergence between MEG and fMRI sensitivities is more likely to manifest itself in such experiments.

Reading is a high-level cognitive task that has been extensively characterized with both MEG and fMRI. For silent single-word reading, there are a considerable number of published reports that, within each imaging modality, show remarkable consistency on the activated areas and their time courses and functional roles (Jobard et al., 2003; Salmelin, 2007). With the increasing number of studies, apparent differences between MEG and fMRI views of reading have started to form a systematic pattern. Although both methods show involvement of the occipital, occipitotemporal, and temporal cortex in reading, the resulting views of those areas' functional roles are partly inconsistent (e.g., Jobard et al., 2003; Vartiainen et al., 2009a). 


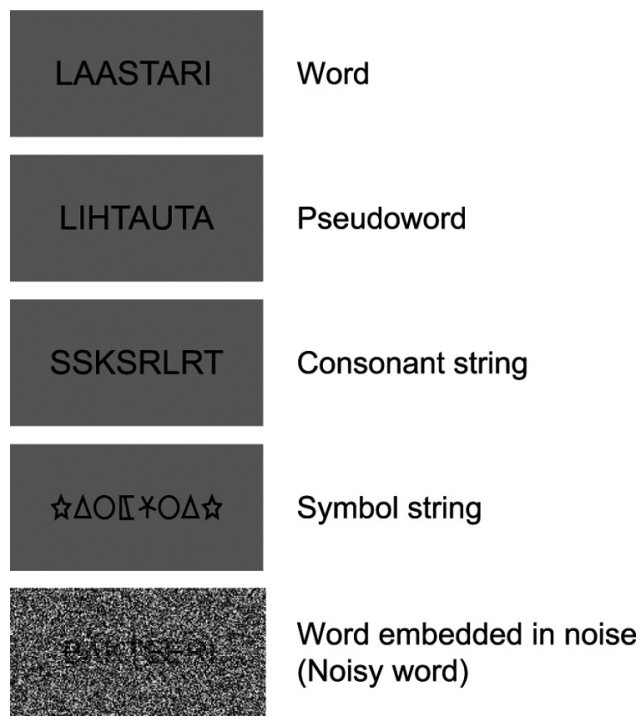

Figure 1. Examples of the stimulus types. An identical paradigm was used in the fMRI-EEG and MEG-EEG measurements. Stimuli were presented in blocks of seven items. In addition, there were target blocks in which one stimulus appeared twice (probability 6\%). On average, every sixth block was a 12 s rest block (gray background).

One possible source of these discrepancies is that the fMRI and MEG studies of reading have typically been conducted using different experimental paradigms and languages, on different participants. Here, we directly compared the fMRI and MEG data of the same individuals performing the same reading task. The paradigm was constructed by merging previous fMRI and MEG reading paradigms. As an additional tool for verification, EEG was measured simultaneously with MEG and fMRI to evaluate whether the subjects performed the task similarly in both environments.

Hemodynamic responses have been suggested to correspond to electromagnetic activation indexed by MEG/EEG oscillatory power, particularly at high frequencies (Foucher et al., 2003; Lachaux et al., 2007; Rosa et al., 2010). This perspective on the hemodynamic correlates of electromagnetic activity derives mostly from low-level perceptual tasks, analysis of spontaneous activity, induced responses, and fMRI-EEG in epilepsy [see SalekHaddadi et al. (2003) and Laufs et al. (2008) for reviews], and contrasts with the strong focus on evoked responses in MEG/EEG cognitive research, including language. In the present study, we compared the analysis approaches that are most commonly used in MEG and fMRI reading experiments, namely, MEG evoked responses and $\mathrm{AMRI}$ BOLD signals.

\section{Materials and Methods}

\section{Subjects and measurement sessions}

Fifteen right-handed, native Finnish-speaking subjects (7 females; age $20-49$ years, mean 27 years) participated in the experiment. The participants gave their informed consent, in agreement with the prior approval of the Helsinki and Uusimaa Ethics Committee.

Simultaneous fMRI-EEG and MEG-EEG were recorded from each participant (at least 1 week apart, order counterbalanced), using an identical experimental paradigm. For two participants, the EEG data in fMRI could not be analyzed due to a technical problem.

\section{Stimuli and experimental design}

The stimuli consisted of Finnish words, pseudowords, consonant strings, symbol strings, and noisy words (embedded in high-frequency visual noise) (Fig. 1). There were 112 stimuli in each category (length 7-8 letters/symbols). Noisy words were constructed by embedding words in high-level noise that made recognition of the underlying word practically impossible (Tarkiainen et al., 1999). Pseudowords were pronounceable letter strings that did not have meaning. Consonant strings were made by randomly selecting 7 to 8 consonants from the real words (any one letter maximally twice in a row). Symbol strings consisted of 10 different symbols that were selected randomly to form 7- and 8-symbol strings (the same symbol maximally twice in a row).

The stimuli were presented visually one at a time (visual angle $<7^{\circ}$ ). To avoid potentially dangerous heating of the EEG electrodes inside the magnet, the measurement time was limited to $30 \mathrm{~min}$. For optimal signal-to-noise ratio, we used a block design with seven stimuli of the same category in each block. Each stimulus was shown for $300 \mathrm{~ms}$, followed by $1200 \mathrm{~ms}$ of gray background. In addition to the five stimulus conditions ( 16 blocks each), there were 16 blocks of rest (12 s of gray background). Additional random jitter (0-600 ms) was included in the beginning of each block to avoid synchronization of the EEG responses with the MRI pulses. Blocks from the different stimulus categories were presented in a partially counterbalanced order, and the stimuli were presented in a random order within the blocks. In MEG and EEG, the response to the first stimulus of each block was excluded from the analysis to avoid rapid attention-related effects.

Additional target blocks (one per stimulus category), with one stimulus appearing twice in a row, were presented to keep the subjects alert. The subject indicated detection with a button press. The target blocks were not included in the analysis.

\section{$M E G$ data acquisition and analysis}

MEG recordings were conducted in a magnetically shielded room using a Elekta Neuromag device (Elekta Oy). The system contains 102 triple sensor elements composed of two orthogonal planar gradiometers and one magnetometer. The gradiometers detect the maximum signal directly above an active cortical area. The signals were bandpass filtered at $0.03-200 \mathrm{~Hz}$ and digitized at $600 \mathrm{~Hz}$. Trials contaminated by eye movements were rejected.

The MEG data were averaged across trials from -0.2 to $1 \mathrm{~s}$ relative to the stimulus onset (mean 75, minimum 42 trials), baseline corrected to the $200 \mathrm{~ms}$ interval immediately preceding the stimulus onset, and low-pass filtered at $40 \mathrm{~Hz}$.

$M E G$ sensor-level analysis. For an initial overview of the data, areal mean signals were calculated over 10 regions: occipital, parietal, and the left and right frontal, rolandic, temporal, and occipitotemporal regions (Vartiainen et al., 2009a). The individual areal mean signals were characterized by measuring the mean amplitude in four time windows: 70 $130 \mathrm{~ms}, 115-150 \mathrm{~ms}, 130-250 \mathrm{~ms}$, and $300-500 \mathrm{~ms}$, that were selected on the basis of the grand-mean and individual signals. Overlapping time windows were selected to capture the short-lasting early responses that showed some interindividual variation in timing.

MEG source analysis: equivalent current dipole modeling. Effects of stimulus type were analyzed by first segregating the sensor-level signals into separable cortical-level spatiotemporal components. The segregation was performed by means of guided current modeling, where the model parameters of each equivalent current dipole (ECD) represent the center of an active cortical patch and the mean orientation and strength of electric current within that area (ECD) (Hämäläinen et al., 1993). The analysis was done with the Elekta Neuromag software package, following standard procedures (Salmelin et al., 1994; Helenius et al., 1998; Hansen et al., 2010). The spatiotemporal components were identified by systematic local changes in the magnetic field. Field patterns persisted over tens to a few hundred milliseconds; ECD model parameters were determined at a time point (width $3.3 \mathrm{~ms}$ ) at which the local field pattern was most dipolar and had least interference from other salient spatiotemporal components. At the selected time point, for the set of MEG sensors that covers the field pattern, the software identifies the sensor that measures the strongest signal and uses a locus below that sensor as the seed point for the ECD model parameter estimation. The location parameter fit is robust against the selection of a seed point in the general region covered by the field pattern. Spatiotemporal components explaining important amounts of variance in the data were included in the multidipole model until at least $75 \%$ of the total variance was explained. Based on this 
criterion, the final models were composed of 11-15 ECDs per subject (mean 14). Thereafter, time courses of activation in those brain areas (source waveforms) were estimated: with the location and orientation parameters now fixed for each ECD in the individual multidipole model, the ECD amplitude parameters were allowed to vary to best account for the measured data in each condition.

For comparison with the fMRI results, the ECD coordinates of the individual subjects were transformed to the Talairach space. For visualization, the ECDs were transformed to the inflated standard template brain Colin27 (Dale and Sereno, 1993).

All individual ECDs were first grouped based on their location parameters (frontal, parietal, temporal, occipitotemporal, and occipital cortex) and the time window within which the amplitude parameter reached the maximum (before vs after $120 \mathrm{~ms}$ ). All clusters that contained an ECD from $>50 \%$ of the individuals (at least 8 of 15 participants) were accepted for further analysis. If several ECDs of the same individual met the criteria for a cluster, only the ECD with the location and peak latency closest to the mean location and peak latency of that cluster was included in further analysis. In the occipital cortex, several ECDs can be identified within the first $120 \mathrm{~ms}$ (cf. Tarkiainen et al., 1999), and the earliest leftand right-hemispheric ECDs of each individual were taken to represent the early visual activations. Thus, each final ECD cluster contained 0-1 ECDs from each individual.

Neural activation within the early time windows was typically transient and was characterized by the maximum level of activation and the time at which the maximum was reached (peak amplitude and peak latency) or, if the signal-to-noise ratio did not allow for reliable definition of the peak values, by the mean amplitude (time window $130-250 \mathrm{~ms}$ ), similarly in all participants. The later, more sustained activation was described by the mean amplitude at 300-500 ms after stimulus. All measures were collected individually for each subject and separately for each condition.

Statistical analysis on the MEG areal mean signals and ECDs. A repeated-measures ANOVA with within-subjects factor stimulus type (words, pseudowords, symbols, consonants, and noisy words) was used to statistically test for significant effects on the strength or timing of the MEG sensor signals and the neural activation in the different cortical areas. When a significant main effect of stimulus type was detected, Bonferroni-corrected pairwise comparisons were used.

MEG source analysis: minimum norm modeling. For visualization, mapping from the MEG sensor signals to cortical activation was additionally investigated using a distributed source model. The analysis was performed using the MNE Suite software package (Hämäläinen, Martinos Center for Biomedical Imaging, Massachusetts General Hospital), which is an implementation of the minimum L2-norm estimate. The measured signals are accounted for by the distribution of electric current that has the minimum overall power. Although minimum norm estimate (MNE) provides a spatially distributed model of the activation sequence, it does not yield direct information about the extent or shape of an active cortical region.
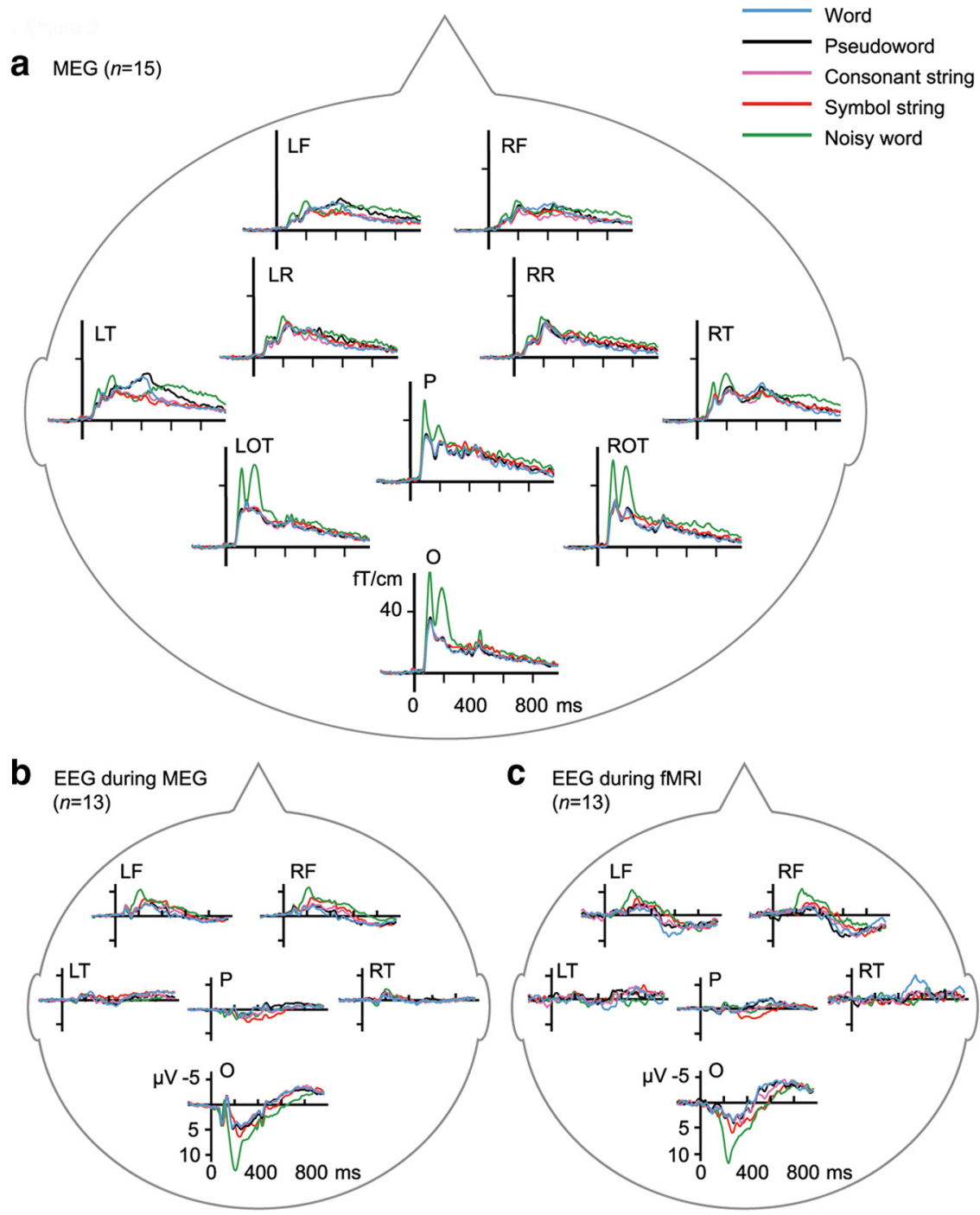

d EEG difference waveforms (fMRI - MEG, $n=13$ )

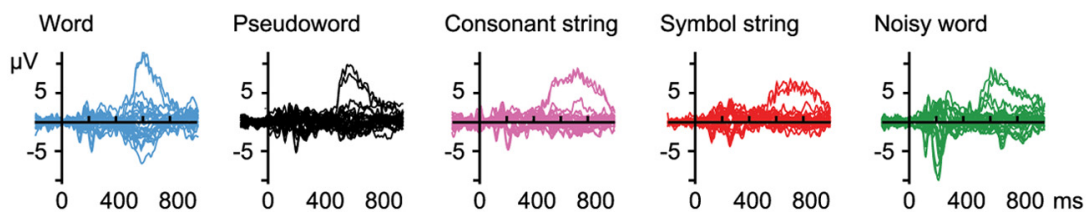

Figure 2. Group-averaged MEG and EEG sensor-level signals. a, MEG areal mean signals calculated over the occipital (0), parietal $(P)$, and left and right occipitotemporal (LOT, ROT), temporal (LT, RT), rolandic (LR, RR), and frontal (LF, RF) cortex, and averaged across 15 participants. The sensor-level areal mean MEG signals were calculated as vector sums; thus, they always have a positive value $(>0) . \boldsymbol{b}$, EEG areal mean signals during the MEG recording calculated across the occipital $(0)$, parietal $(P)$, and left and right temporal (LT, RT) and frontal (LF, RF) electrodes, and averaged across 13 participants. Negative values of the electric potential are plotted upwards. $c$, EEG areal mean signals during the fMRI recording. $\boldsymbol{d}$, EEG difference waveforms. The curves show EEG during MEG minus EEG during $\mathrm{AMRI}$, averaged across 13 participants. For each stimulus type, the difference waveforms for the 28 EEG electrodes are overlaid. A salient difference between the two EEG recordings at $\sim 500-800 \mathrm{~ms}$ is due to an eye-movement artifact in the two most frontal electrodes (FP1-2) that could not be fully removed from the EEG recorded during fMRI.

The cortical surface of each participant was reconstructed from the MRI of that subject using the Freesurfer software (Dale and Sereno, 1993; Fischl et al., 1999). The cortical surface of each hemisphere was covered with $\sim 5000$ potential source locations. The currents normal to the cortical surface were favored over the transverse currents (Lin et al., 2006). The bias of the MNE toward superficial currents was reduced by depth weighting.

Noise-normalized MNEs [dynamical statistical parametric maps (dSPMs)] provide an estimate of the signal-to-noise ratio for each po- 

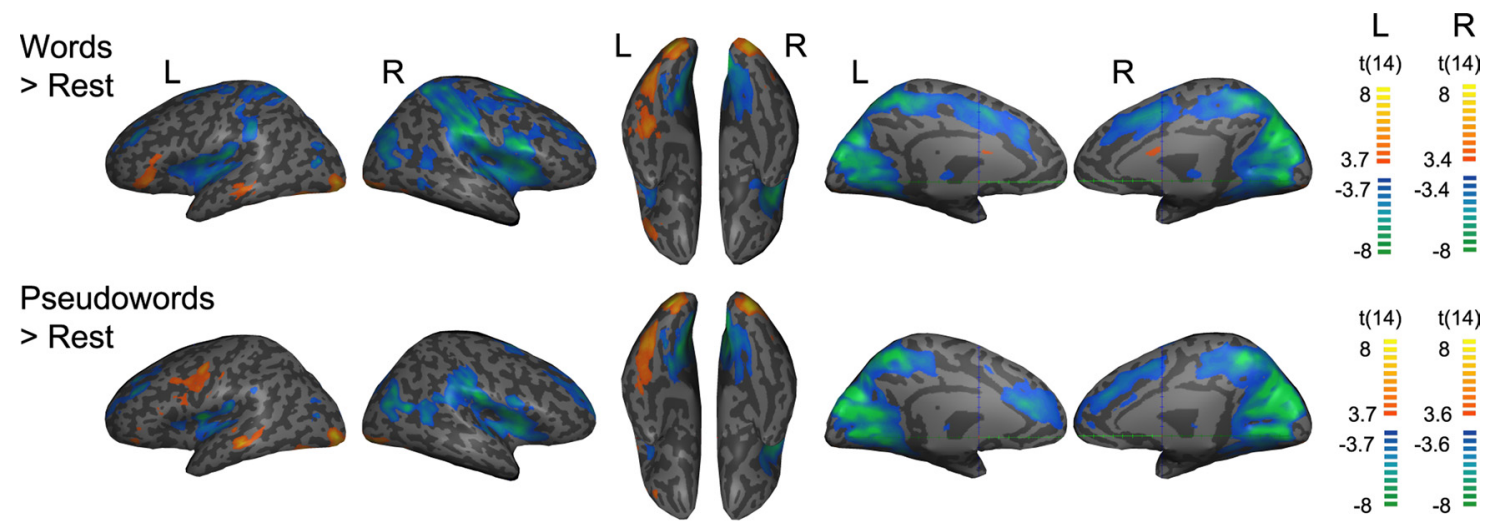

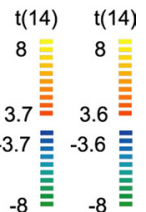
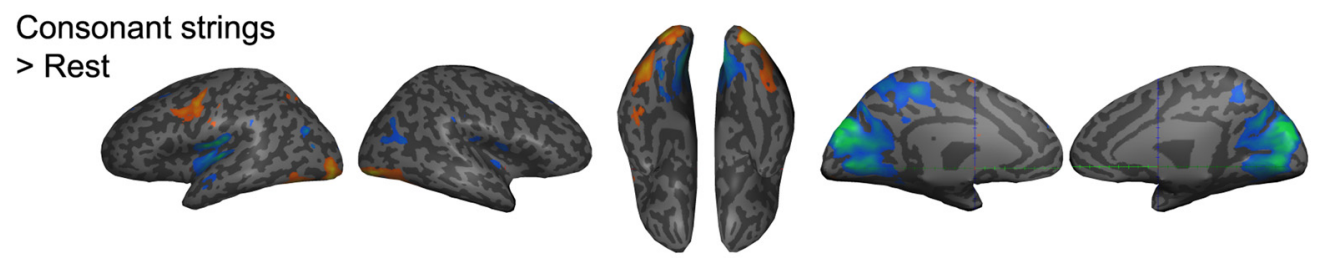

$\mathrm{t}(14) \quad \mathrm{t}(14)$
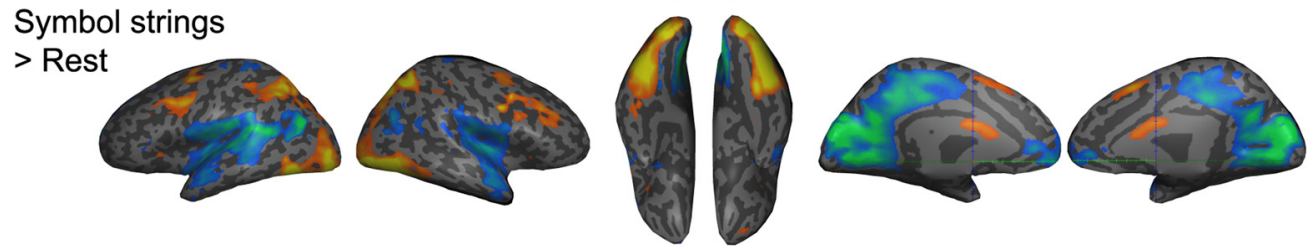

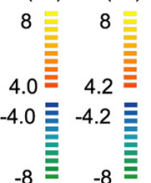
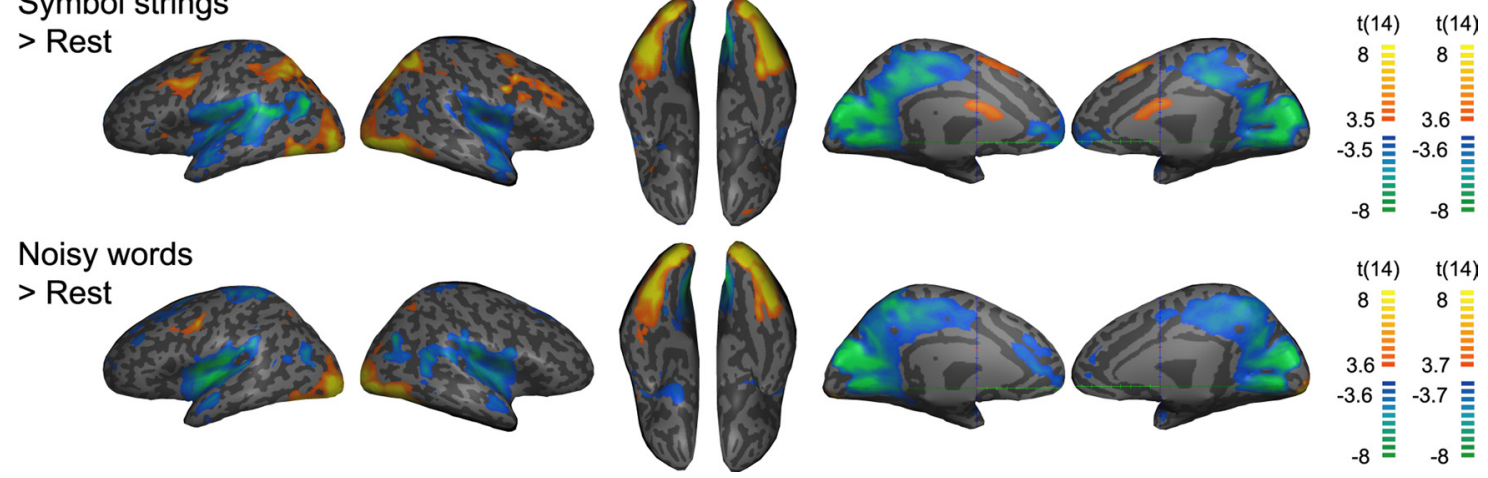

Figure 3. Group-averaged fMRI BOLD activations for the different stimulus types. FMRI statistical parametric maps were overlaid on the surface of the Colin27 template brain (L, left hemisphere; $\mathrm{R}$, right hemisphere) and thresholded at $q(\mathrm{FDR})<0.01$. For the inflated surfaces, the FDR correction was calculated separately in the two hemispheres. Lateral views on the left, ventral views in the middle, and medial views on the right.

tential source location (Dale et al., 2000). A noise covariance matrix was estimated from the $200 \mathrm{~ms}$ prestimulus baseline periods of the unaveraged data. The individual dSPM maps were normalized and the cortical surface of each subject was morphed to the Colin27 brain surface. Thereafter, the dSPMs were averaged across subjects.

\section{fMRI data acquisition and analysis}

MR images were acquired with a 3 Tesla Signa EXCITE MRI scanner (GE Healthcare). The functional data were obtained using a single-shot GREEPI sequence with in-plane resolution $3.4 \times 3.4 \mathrm{~mm}^{2}$ (TR $2.4 \mathrm{~s}$, TE 32 ms, flip angle $75^{\circ}$, acquisition matrix size $64 \times 64$, FOV $22 \mathrm{~cm}$, slice thickness $3 \mathrm{~mm}$ ). Anatomical images were acquired using a T1-weighted 3 D SPGR sequence with $0.9 \times 0.9 \times 1.0 \mathrm{~mm}^{3}$ resolution.

Data were analyzed with standard preprocessing and statistical methods using BrainVoyager QX software (Brain Innovation). During the preprocessing, the functional data were corrected for head movements, and slice scan time correction was applied. A high-pass filter with a 0.01 $\mathrm{Hz}$ cutoff frequency and linear trend removal were used. Serial correlations were compensated for by using a first-order autoregressive model. The anatomical and functional data of each participant were coregistered and transformed to the Talairach space. For group-level fMRI analysis, the data were spatially smoothed with an $8 \mathrm{~mm}$ full width at half maximum Gaussian kernel and normalized.

Statistical analysis was performed within the GLM framework. Regressors for the five stimulus types were convolved with a two-gamma canonical hemodynamic response function. Each stimulus type was contrasted to rest, and contrasts comparing the task conditions were evaluated. At the group level, a random-effects analysis was performed, with separate predictors for each subject. A false discovery rate correction for multiple comparisons (FDR) (Genovese et al., 2002) was calculated separately for each contrast, with the threshold at $q(\mathrm{FDR})<0.01$ for the task versus rest comparisons and $q(\mathrm{FDR})<0.05$ for the task versus task comparisons. Additionally, for definition of activation maxima, a minimum cluster size was determined using an iterative method (Goebel et al., 2006). Anatomical regions were identified using a brain atlas (Duvernoy, 1999). For visualization, the group-level fMRI data were overlaid on the Colin27 template brain.

For comparison with MEG mean activation coordinates, local fMRI activation maxima were additionally determined by heightening the statistical threshold until the clusters of activation in the different brain lobes and in the two hemispheres became separable. fMRI signal change (as a percentage) was measured, for each stimulus category, in $10 \times 10 \times$ $10 \mathrm{~mm}^{3}$ ROIs centered at these fMRI activation maxima and at the mean MEG coordinates. Previous fMRI experiments (Cohen et al., 2002; Vinckier et al., 2007) have detected activation to letter strings in the left occipitotemporal cortex (LOTC), slightly anterior to the area where MEG shows equal activation for all letter strings at $\sim 150 \mathrm{~ms}$ after the word onset (Tarkiainen et al., 1999, 2002; Cornelissen et al., 2003). In this so-called visual word form area (VWFA), the BOLD signal is stronger to real words than to consonant strings. To compare fMRI and MEG activation of the same individuals in this region, we selected an additional ROI centered at the peak of the assumed VWFA (Vinckier et al., 2007). For the ROIs determined independently of the present fMRI data (MEG mean coordinates and assumed VWFA), the effects of stimulus type on the fMRI signal change were tested using a repeated-measures 
ANOVA with within-subjects factor stimulus type and, when a significant main effect was detected, with Bonferroni-corrected pairwise comparisons.

\section{EEG data acquisition and analysis}

In fMRI, EEG data were acquired using a 32channel customized cap (BrainCap MR). The signals were bandpass filtered at 0.016-1000 $\mathrm{Hz}$ and sampled at $5000 \mathrm{~Hz}$. The induced EPI artifacts in EEG recordings were removed (Koskinen and Vartiainen, 2009) and the data resampled to $200 \mathrm{~Hz}$. In MEG, EEG data were recorded using a 64-channel cap (Elekta Neuromag), bandpass filtered at $0.03-200 \mathrm{~Hz}$, and digitized at $600 \mathrm{~Hz}$.

For EEG comparison in MEG and fMRI, a corresponding set of 28 EEG electrodes was analyzed, using an average reference (Dien, 1998). Trials contaminated by eye movements were excluded. The EEG trials were averaged from -0.2 to $1 \mathrm{~s}$ relative to the stimulus onset, baseline corrected to the $200 \mathrm{~ms}$ interval immediately preceding the stimulus onset, and low-pass filtered at $40 \mathrm{~Hz}$.

The EEG signals were visualized by calculating areal mean signals across six sensor groups (occipital, parietal, left and right temporal, and left and right frontal), separately for each individual and, thereafter, averaged across individuals.

The mean amplitude in three time windows, $70-130 \mathrm{~ms}$ (first positive response), $115-155 \mathrm{~ms}$ (first negative response), and 130-500 ms (second positive response), selected on the basis of the grand-mean and individual signals, were tested using a repeated-measures ANOVA with within-subjects factors modality (fMRI and MEG) and stimulus type. When a significant main effect of stimulus type was detected, Bonferroni-corrected pairwise comparisons were used.

\section{Results}

MEG and EEG: sensor-level signals

Figure 2 displays the MEG and EEG sensorlevel areal mean signals. In line with previous MEG studies of reading (Salmelin, 2007), responses to letter strings [words (W), pseudowords (P), and consonant strings (C)], symbol strings (S), and words embedded in noise [referred to as noisy words $(\mathrm{N})$ in the following text] differed over the occipital, occipitotemporal, and temporal cortex.

Each visual stimulus elicited an MEG response over the occipital region at $\sim 100 \mathrm{~ms}$ after the stimulus onset. This occipital response was stronger to noisy words than to the other stimulus types; see supplemental Table S1 (available at www.jneurosci.org as supplemental material) for the statistical tests. For the noisy words, the occipital MEG response was followed by a separate response over the occipital and occipitotemporal regions at $\sim 200 \mathrm{~ms}$.

In line with previous MEG studies, letter and symbol strings elicited a bilateral response that peaked over the left and right occipitotemporal sensors at $\sim 150 \mathrm{~ms}$ and $\sim 200 \mathrm{~ms}$, respectively. The response appeared stronger to letter than to symbol strings over the left hemisphere (LH); this effect did not reach statistical significance in the sensor-level analysis, but see source-level MEG results below.
$\operatorname{MEG}(n=15)$

a Left occipital cortex at $\sim 100 \mathrm{~ms}(\mathrm{n}=14)$

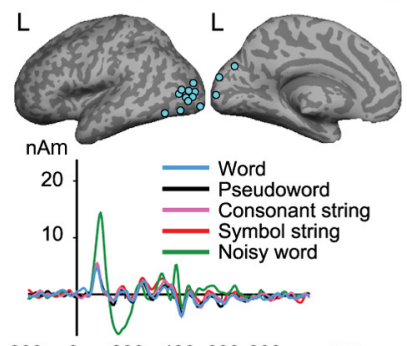

Right occipital cortex at $\sim 100 \mathrm{~ms}(\mathrm{n}=15)$

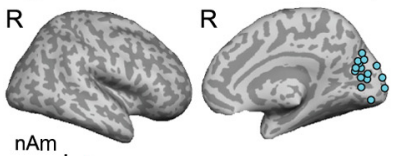

20

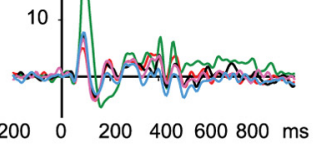

b MNE Noisy words $65-115 \mathrm{~ms}$

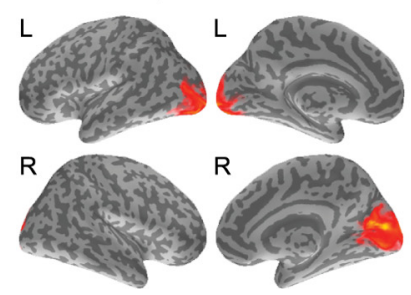

C Left occipital cortex, TAL $-21,-92,-5$
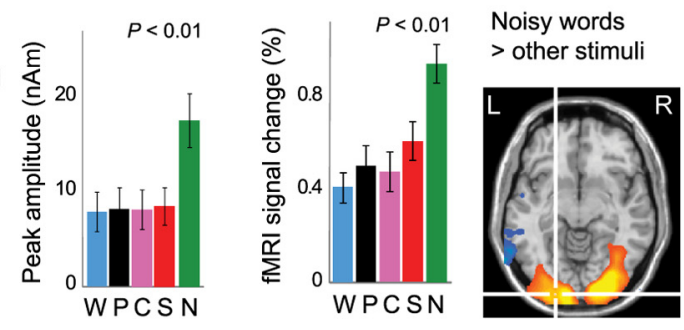

Right occipital cortex, TAL 13, -90, 4

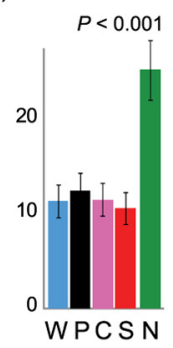
$P<0.001$

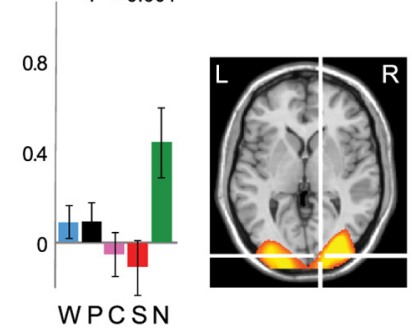

d Noisy words > other stimuli

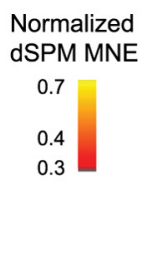

fMRI $(n=15)$
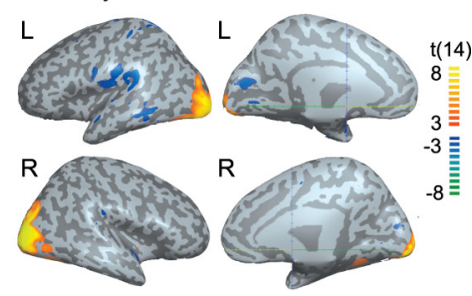

Figure 4. MEG and fMRI activations and stimulus effects in the occipital cortex. $\boldsymbol{a}$, MEG activation in the left (above) and right (below) occipital cortex at $\sim 100 \mathrm{~ms}$. The colored dots indicate the individual centers of the active brain region (lateral and medial views). The same ECDs also accounted for part of the activation evoked by noisy words at $\sim 200 \mathrm{~ms}$. The curves show the mean time of activation in each region and the bar graphs the ECD maximum activation values ( $\pm S E M)$ for the five stimulus types (1) percentage) in the $10 \times 10 \times 10 \mathrm{~mm}^{3}$ ROls centered at the mean MEG coordinate in the left and right occipital cortex ( $P$ values refer to the ANOVA). The locations of the ROI center voxels are indicated by a white crosshair on the fMRI contrast image noisy words $>$ other stimuli $[q(F D R)<0.05]$. $\boldsymbol{d}$, The surface display gives a more complete view of the group-averaged fMRI BOLD activations in the contrast noisy words $>$ other stimuli. The fMRI statistical parametric maps were overlaid on the Colin 27 template brain $[q(\mathrm{FDR})<0.05]$. nAm, nanoampere-meters.

Words and pseudowords elicited a sustained MEG response that reached the maximum at $\sim 400 \mathrm{~ms}$ after the stimulus onset over the left temporal cortex (N400/N400m response in EEG/ MEG literature). This response was stronger to words and pseudowords than to the other stimulus types. A sustained response was detected over the right temporal cortex as well, but the stimulus effects were less pronounced than over the left temporal cortex.

The EEG displayed, overall, the same response patterns and effects as MEG, and those effects were detected similarly during the MEG and fMRI recordings; see supplemental Table S2 (available at www.jneurosci.org as supplemental material). The first positive EEG response (P1) was detected at $\sim 100 \mathrm{~ms}$, mainly in the occipital electrodes, followed by strong responses specifically to noisy words at $\sim 200-250 \mathrm{~ms}$, corresponding to the early occipital effects in the MEG sensors. The first negative EEG response at $\sim 150 \mathrm{~ms}(\mathrm{~N} 1 / \mathrm{N} 170)$, visible in the occipital electrodes, 
$\operatorname{MEG}(n=15)$

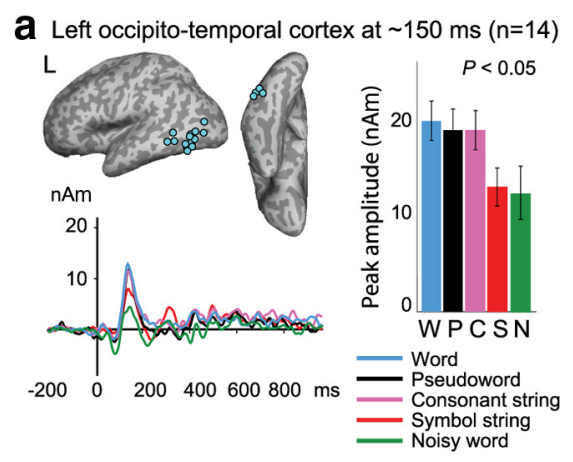

Right occipito-temporal cortex at $\sim 200 \mathrm{~ms}(\mathrm{n}=9)$
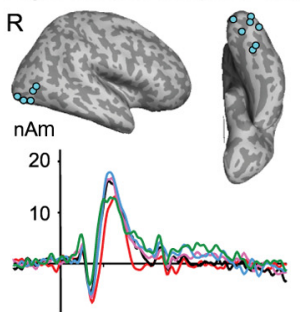

$-200 \quad 0 \quad 200400600800 \mathrm{~ms}$

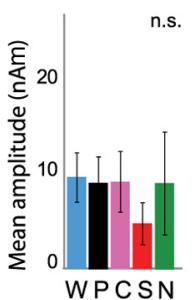

b MNE 145-195 ms
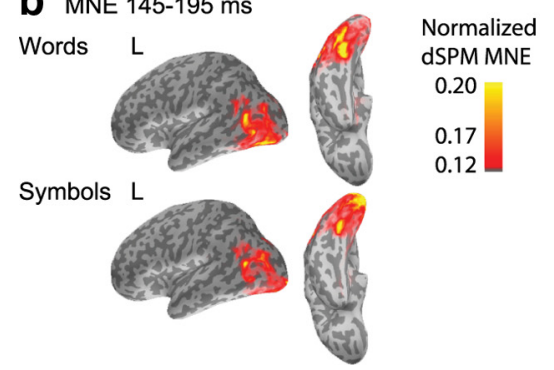

fMRI $(n=15)$

C ROI at MEG mean activation, TAL -42, -71, -10

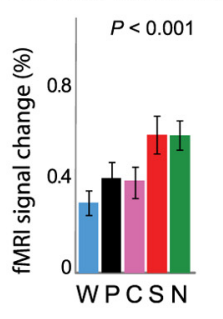

Letter strings

$>$ Symbol strings

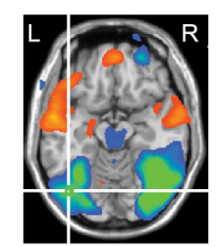

Assumed WWFA, TAL $-43,-57,-12$

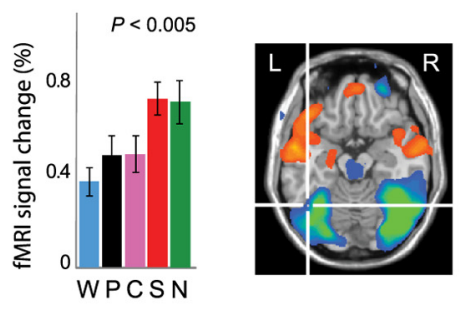

d Letter strings $>$ Symbol strings

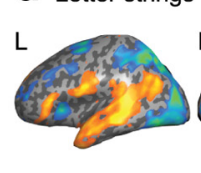

e Words

$>$ Rest

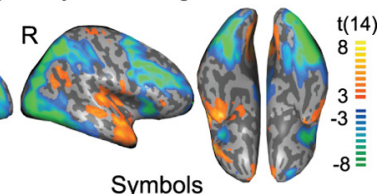

Symbols

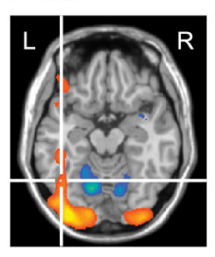

$>$ Rest

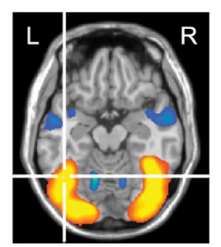

Figure 5. MEG and fMRI activations and stimulus effects in the occipitotemporal cortex. $\boldsymbol{a}$, MEG activation in the left occipitotemporal cortex at $\sim 150 \mathrm{~ms}$ (above) and in the right occipitotemporal cortex at $\sim 200 \mathrm{~ms}$ (below), with the mean time course of activation. The upper bar graph displays the ECD maximum activation values ( \pm SEM) for the left occipitotemporal activation showing a significant stimulus effect for letter $>$ symbol strings. The lower bar graph displays the ECD mean amplitude in the time window 130-250 ms for the right occipitotemporal activation (no stimulus effects). $\boldsymbol{b}$, The MNE dSPM maps depict the mean left-hemisphere occipitotemporal activation in the time window 145-195 ms for words and symbols. See also supplemental Figure S1 (available at www.jneurosci.org as supplemental material). c, The bar graphs show the fMRI signal change (as a percentage) in two left-hemisphere $10 \times 10 \times 10 \mathrm{~mm}^{3}$ ROls centered at the mean MEG coordinate in the left inferior occipitotemporal cortex (above) and in the assumed VWFA (Vinckier et al., 2007; below). The location of each ROI center voxel is indicated by a white crosshair on the fMRI contrast image letter $>$ symbol strings $[q(\mathrm{FDR})<0.05]$. $\boldsymbol{d}$, The surface display gives a more complete view of the group-averaged fMRI BOLD activations in the contrast letter $>$ symbol strings $[q(\mathrm{FDR})<0.05]$. $\boldsymbol{e}$, fMRI BOLD activation in the contrasts words $>$ rest and symbols $>$ rest $[q(F D R)<0.01]$. The white crosshair indicates the location of the assumed VWFA. nAm, nanoampere-meters.

corresponded to the simultaneous letter-string effect in MEG; in the EEG data, the effect reached significance for the consonants and pseudowords $>$ symbols (occipital electrodes at 115-150 $\mathrm{ms}$ ). An effect corresponding to the sustained $\mathrm{N} 400 \mathrm{~m}$ response in MEG was most clearly visible in the parietal EEG electrodes at $\sim 400$ ms. Importantly, the EEG data did not indicate neurophysiological differences that could explain any potential incompatibilities between the MEG and PMRI results.

At individual EEG electrodes, some differences between the two EEG sessions were visible (Fig. $2 d$ ). In the difference waveforms, the most prominent feature is the eye-movement artifact that could not be fully removed from the EEG recorded during fMRI; it shows at the two most frontal electrodes (FP1 and FP2). This difference did not reach significance in the statistical tests (supplemental Table S2, available at www.jneurosci.org as sup- plemental material). With close inspection, the early responses at $\sim 100-200 \mathrm{~ms}$, which are more salient in the EEG recorded during MEG (probably due to better EEG signalto-noise ratio in the MEG than fMRI environment), can also be observed in the difference waveform (cf. supplemental Table S2, available at www.jneurosci.org as supplemental material, significant main effect of modality at 70-130 ms). Furthermore, the EEG responses to noisy words at $\sim 200 \mathrm{~ms}$ appear to be stronger at some electrodes when measured during MEG than during fMRI, but this difference was not statistically significant.

\section{MEG: sequence of activation at the source level}

The source-level analysis illustrated in Figures $4-7$ shows the active areas that were found consistently (in at least 8 subjects) and their mean time course of activation, as modeled by ECDs, and noise-normalized MNE dSPMs; see supplemental Figure S1 (available at www. jneurosci.org as supplemental material) for MNEs to all stimulus categories. Both ECD and MNE analysis of the MEG data revealed the typical sequence of activation in response to visual stimuli and written words. Activation was first detected in the occipital cortex at $\sim 100 \mathrm{~ms}$ (see Fig. 4 ), then in the occipitotemporal cortex at $\sim 150-200 \mathrm{~ms}$ (see Fig. 5), and thereafter, in the left and right superior temporal cortex, and the left parietal and frontal cortex (see Figs. 6, 7). The mean Talairach coordinates and anatomical regions for the centers of the consistently activated regions are listed in supplemental Table S3 (available at www.jneurosci.org as supplemental material).

\section{fMRI: BOLD activations}

The fMRI data showed stronger activation in task than rest miniblocks in a set of occipital, inferior occipitotemporal, temporal, parietal, and frontal areas that are typically involved in visual processing and reading tasks (Fig. 3; supplemental Table S4, available at www.jneurosci.org as supplemental material). An overview of the significant activations in the contrasts between the five different stimulus types is presented in Figures $4-7$, and the activation maxima are listed in supplemental Table S5 (available at www.jneurosci.org as supplemental material).

\section{MEG versus fMRI: occipital cortex}

Activation in the occipital cortex was detected similarly with MEG and fMRI (Fig. 4). In MEG, several occipital sources were usually identified within the first $120 \mathrm{~ms}$ after each stimulus; the first left-hemispheric and right-hemispheric ECDs of each individual are depicted in Figure 4. This bilateral activation was 
MEG $(n=15)$

a MEG activations at $300-500 \mathrm{~ms}$
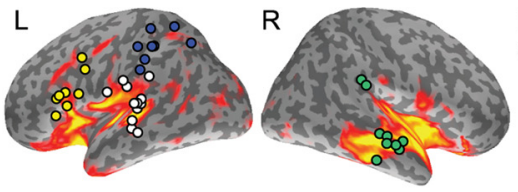

Normalized dSPM MNE

0.40

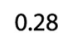

0.22

\section{b Temporal cortex at $\sim 250-700 \mathrm{~ms}$}

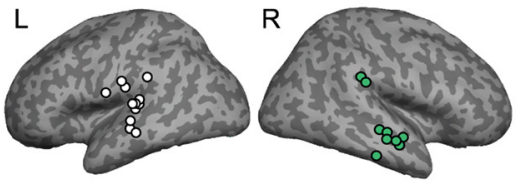

- Left temporal cortex $(n=12)$

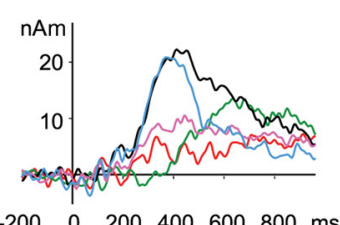

$-200 \quad 0 \quad 200400600800 \mathrm{~ms}$

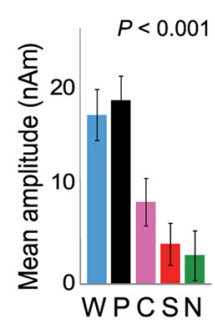

- Right temporal cortex $(n=9)$
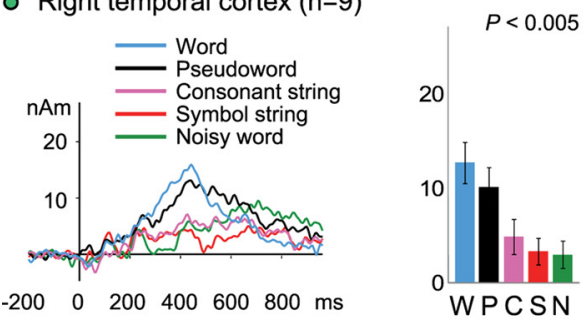

fMRI $(n=15)$

C Words and Pseudowords $>$ Consonants
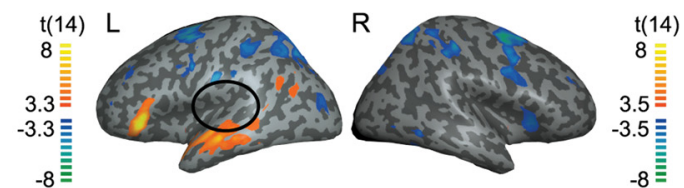

Letter strings $>$ Symbols and Noisy words
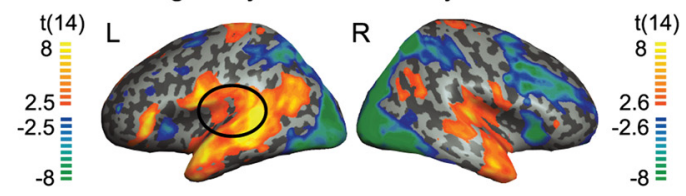

d ROI at MEG mean activation

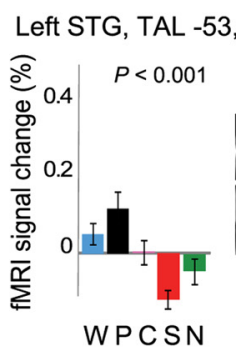

$-25,5$

W, $\mathrm{P}>\mathrm{C}$
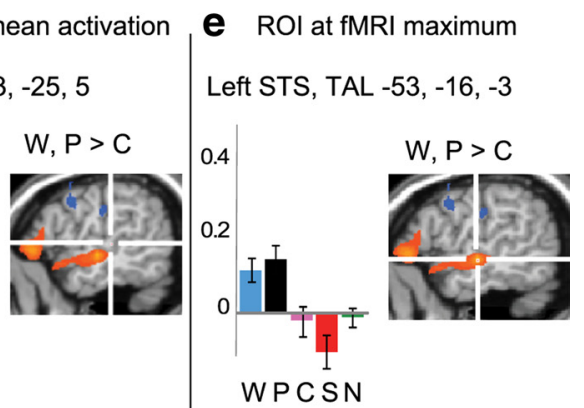

Right STG,TAL 55, -18, 5

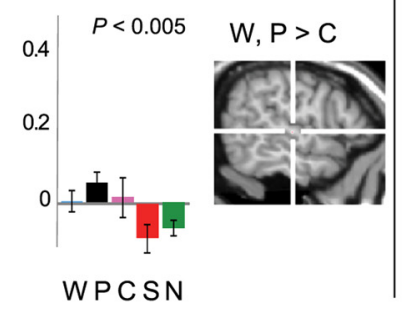

Right TTG,TAL 49, -24, 12

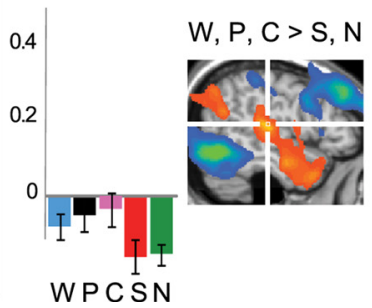

Figure 6. MEG and fMRl activations in the anterior brain regions and stimulus effects in the temporal cortex. $\boldsymbol{a}, \mathrm{MEG}$ activations from $\sim 250 \mathrm{~ms}$ onwards. The ECDs are overlaid on the mean MNE dSPM map for words at $300-500$ ms. The ECDs are color coded by region (left temporal: white; frontal: yellow; parietal: blue; right temporal: green). $\boldsymbol{b}$, MEG stimulus effects in the temporal cortex. The curves show the mean time course of activation for each ECD cluster and the bar graphs the ECD mean amplitude values ( \pm SEM) in the time window $300-500 \mathrm{~ms}$. c, The surface displays gives an overview of the group-averaged fMRI BOLD activations in the contrasts words and pseudowords $>$ consonants and letter strings $>$ symbols and noisy words [ $q(F D R)<0.05]$. The black ellipse indicates the approximate location of the left temporal MEG activation. $\boldsymbol{d}$, The bar graphs display the fMRI signal change (as a percentage) in the ROIs centered at the mean MEG coordinates in the left (above) and right (below) temporal cortex. The location of the center voxel is indicated on the fMRI contrast image words and pseudowords $>$ consonants $[q(F D R)<0.05]$. e, The bar graphs display the fMRI signal change (as a percentage) in the ROls centered at the fMRI activation maxima in the left temporal cortex (above; words and pseudowords $>$ consonants) and in the right temporal cortex (below; letter strings $>$ other stimuli). The fMRI activation maxima were derived from the relevant contrast shown in the fMRI contrast image. STS, Superior temporal sulcus; TTG, transverse temporal gyrus; nAm, nanoampere-meters.

strongest to noisy words; see supplemental Table S6 (available at www.jneurosci.org as supplemental material) for the statistical tests. From $\sim 150$ ms onwards, the MEG sequence to noisy words diverged from that to the other stimulus types. For the noisy words, the bilateral occipital source regions were active again at $\sim 200 \mathrm{~ms}$, with opposite direction of current flow (i.e., opposite polarity of the source waveforms).

Similarly, in fMRI, the occipital cortex was activated bilaterally in response to all types of visual stimuli, and this activation was enhanced for noisy words as compared with the other stimulus types. The mean MEG center of activation (cf. supplemental Table S3, available at www.jneurosci.org as supplemental material) was located $8 \mathrm{~mm}$ apart from the $\mathrm{fMRI}$ activation maximum in the $\mathrm{LH}$ and $21 \mathrm{~mm}$ apart in the right hemisphere (RH) but fell, in both hemispheres, within the fairly extended region that showed the same effect noisy words $>$ other stimuli in fMRI [fMRI region of interest (ROI) centered at the MEG mean coordinate, $\mathrm{LH}: \mathrm{N}>\mathrm{S}, \mathrm{C}, \mathrm{P}, \mathrm{W}$; RH: N > S, C, P]; see supplemental
Table S7 (available at www.jneurosci.org as supplemental material) for the statistical tests.

\section{MEG versus fMRI: occipitotemporal cortex}

Activation in the LOTC was detected with both MEG and PMRI (Fig. 5), but with opposite stimulus effects for letter versus symbol strings. MEG showed activation in the LOTC at $\sim 150 \mathrm{~ms}$ to words, pseudowords, consonants, and symbols, and this activation was stronger to letter than symbol strings. The mean center of activation was detected in the left temporo-occipital incisure, between the fusiform gyrus and the inferior temporal gyrus.

The fMRI data showed the opposite effect. Responses to symbol strings were enhanced as compared with letter strings throughout the LOTC, including the regions where the MEG activation was located [ $\mathrm{fMRI}$ ROI at the MEG mean coordinate: $\mathrm{S}>\mathrm{C}, \mathrm{P}, \mathrm{W}]$. The mean MEG center of activation and the fMRI activation maximum (symbol $>$ letter strings) were located 10 $\mathrm{mm}$ apart. In fMRI, the enhanced activation to symbol versus 


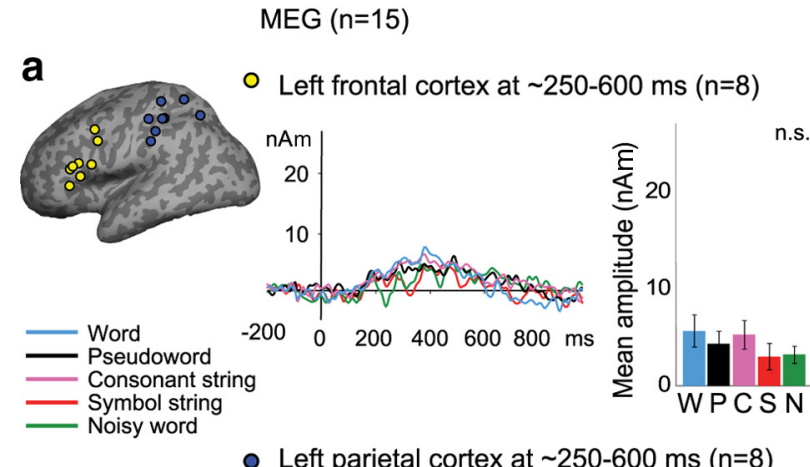

- Left parietal cortex at $\sim 250-600 \mathrm{~ms}(\mathrm{n}=8)$
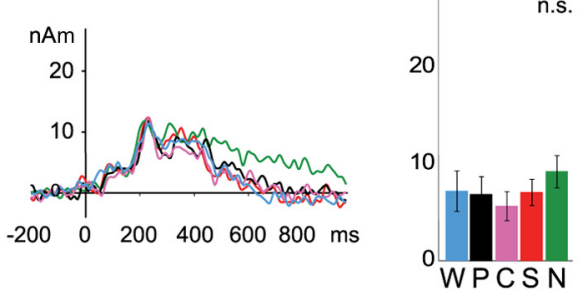

fMRI $(n=15)$
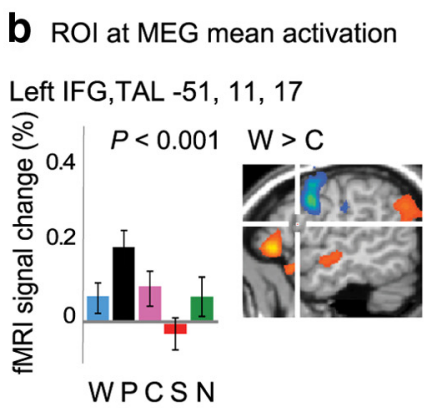

Left IPL,TAL -43, $-47,43$

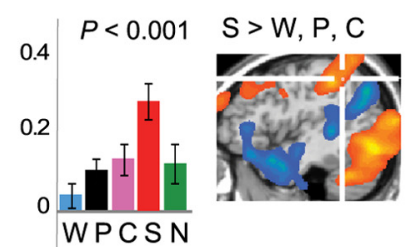

\section{ROI at fMRI maximum}

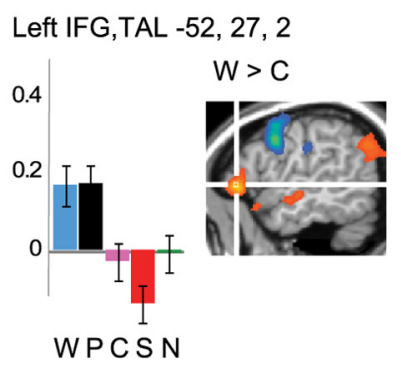

Left IPL, TAL -37, -53, 46

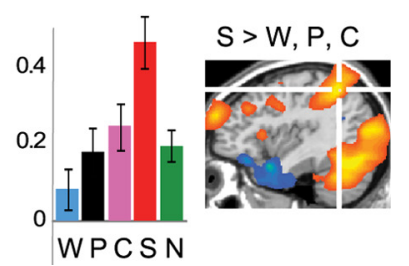

Figure 7. MEG and fMRI stimulus effects in the frontal and parietal cortex. $a$, MEG activation in the left frontal and parietal cortex from $\sim 250 \mathrm{~ms}$ onwards (see Fig. $6 \mathrm{~A}$ for the corresponding MNE map). The curves show the mean time course of activation for each ECD cluster and the bar graphs the ECD mean amplitude values ( \pm SEM) in the time window $300-500$ ms (n.S., nonsignificant effect of stimulus type in the ANOVA). $\boldsymbol{b}$, The bar graphs display the fMRI signal change (as a percentage) in the ROIs centered at the mean MEG coordinates in the left frontal (above) and parietal (below) cortex. The locations of the center voxels are indicated on the fMRI contrast images words $>$ consonants and symbol $>$ letter strings $[q(\mathrm{FDR})<0.05]$. c, The bar graphs display the fMRI signal change (as a percentage) in the ROIs centered at the fMRI activation maxima in the left frontal cortex (above; words $>$ consonants) and in the left parietal cortex (below; symbol $>$ letter strings). IFG, Inferior frontal gyrus; IPL, inferior parietal lobule; nAm, nanoampere-meters.

letter strings extended to the peak of the assumed VWFA [TAL $-42,-57,-12$ (Vinckier et al., 2007); fMRI ROI at the assumed VWFA: $\mathrm{S}>\mathrm{W}$ ]. No differences between words and consonants or between pseudowords and consonants were detected in the left occipitotemporal cortex using either MEG or fMRI.

MEG activation in the right occipitotemporal cortex was found at $\sim 200 \mathrm{~ms}$ after each stimulus (Fig. 5). This activation did not show significant stimulus effects. In fMRI, increased right occipitotemporal activation was detected in response to symbols and noisy words. The mean MEG center of activation and the fMRI activation maximum (symbol $>$ letter strings) were located $8 \mathrm{~mm}$ apart.

\section{MEG versus fMRI: temporal cortex}

Bilateral activation in the temporal cortex was detected with both MEG and fMRI but with partly different stimulus effects and localization (Fig. 6). Strong sustained MEG activation to words and pseudowords was observed in the left and right temporal cortex from $\sim 250 \mathrm{~ms}$ onwards, with the location, orientation of current flow, and time course of typical N400m sources (Simos et al., 1997; Helenius et al., 1998). In both hemispheres, the mean MEG center of activation was located in the middle part of the superior temporal gyrus (STG). In fMRI, at the same location bilaterally, activation was detected to letter versus symbol strings (fMRI ROI at the MEG mean coordinate; $\mathrm{LH}: \mathrm{W}, \mathrm{P}>\mathrm{S}$ and $\mathrm{P}>$ $\mathrm{N} ; \mathrm{RH}: \mathrm{P}>\mathrm{N}, \mathrm{S})$.

In fMRI, an MEG-comparable effect of words and pseudowords $>$ consonants was detected in the LH, in the superior temporal sulcus, $12 \mathrm{~mm}$ anterior and inferior to the MEG mean locus, and this fMRI activation extended for $\sim 3.5 \mathrm{~cm}$ toward the temporal pole. In the $\mathrm{RH}$, the mean center of MEG activation (words/pseudowords $>$ consonants) was located 11 $\mathrm{mm}$ anterior-inferior to the fMRI activation maximum (letter strings $>$ other stimuli).
In MEG, the MNEs additionally suggested activation to words and pseudowords in the insula bilaterally, accompanied by activation in the anterior medial surfaces of the brain. However, the spatial resolution of MEG in these deep cortical areas is poor $(\sim 4$ $\mathrm{cm}$ ) (Molins et al., 2008), and these activation patterns carry a strong resemblance to those elicited by eye blinks; therefore, they were not included in the further analysis.

\section{MEG versus fMRI: frontal cortex}

Activation in the left frontal cortex was detected with both methods, but only the fMRI responses showed differences between the stimulus types (Fig. 7). fMRI activation in the left inferior frontal gyrus (LIFG) was enhanced to words and pseudowords versus symbols and noisy words; among the letter strings, the LIFG activation was stronger to words than consonants. In MEG, frontal activation was identified in 8 of 15 participants, on average 22 $\mathrm{mm}$ posterior to the fMRI activation maximum. The MEG activation was fairly weak and did not show any stimulus effects. In fMRI, activation in this more posterior part of the LIFG was stronger to pseudowords than to noisy words, symbols, or words (fMRI ROI at the MEG mean coordinate: $\mathrm{P}>\mathrm{N}, \mathrm{S}, \mathrm{W}$ ).

\section{MEG versus fMRI: parietal cortex}

In the parietal cortex, MEG and fMRI showed different functionality and lateralization (Fig. 7). MEG activation was detected in the left parietal cortex at $\sim 250-600$ ms after each stimulus, with no stimulus effects. In fMRI, parietal activation was detected bilaterally and it was stronger to symbols than to letter strings ( $\mathrm{fMRI}$ ROI at the MEG mean coordinate: $\mathrm{S}>\mathrm{W}, \mathrm{P}$ ). The MEG mean center of activation was located in the inferior parietal lobule, $9 \mathrm{~mm}$ apart from the fMRI activation maximum (symbol > letter strings). 


\section{Discussion}

This study compared neural activation, as reflected in MEG evoked responses and fMRI BOLD, directly in the same subjects in a well studied cognitive paradigm, silent single-word reading. We found that these commonly used measures of neural activity showed involvement of largely comparable brain regions in reading, but with marked differences in their functional roles. The simultaneously recorded EEG responses did not indicate neurophysiological differences between the MEG and fMRI measurement sessions that could account for the observed incompatible functional patterns. Instead, the differences are likely to reflect actual dissimilarities in the way neural activity in a high-level cognitive task is picked up by MEG evoked responses and fMRI BOLD.

The experiment was designed as a merger of previous MEG (Tarkiainen et al., 1999; Cornelissen et al., 2003) and fMRI (Cohen et al., 2000, 2003) studies of reading. Those earlier, separate findings were, reassuringly, reproduced in this combined MEG-fMRI-EEG study. The MEG data showed occipital activation at $\sim 100 \mathrm{~ms}$ that was stronger to words embedded in noise than to clearly visible words, similarly to the activation previously identified as visual feature analysis (Tarkiainen et al., 1999). The subsequent activation in the LOTC at $\sim 150 \mathrm{~ms}$ also replicated the behavior previously identified as reflecting letterstring analysis (Tarkiainen et al., 1999; Cornelissen et al., 2003): the responses were equally strong to clearly visible letter strings, regardless of their lexicality (words, pseudowords, consonants), and weaker to symbols. The superior temporal responses at 200-800 ms were stronger to words and pseudowords than to consonants bilaterally, in agreement with earlier MEG studies that have consistently associated activation of this region with semantic, phonological, and morphological processing of written words and pseudowords (Helenius et al., 1998; Marinkovic et al., 2003; Pylkkänen et al., 2006; Vartiainen et al., 2009a,b).

The occipital fMRI activation, particularly strong to noisy words, agrees with the fMRI view of that area's role in the analysis of visual properties (Jobard et al., 2003). The stronger LOTC activation to symbol than letter strings is in accordance with previous fMRI data (Brem et al., 2009), and the equal fMRI activation to words and consonant strings is in line with earlier fMRI studies that have used similar tasks (Tagamets et al., 2000; Cohen et al., 2003). The enhanced activation to real words and pseudowords in the LIFG and left middle temporal gyrus agrees with previous fMRI studies that have associated activation of these regions with semantic analysis and the graphophonological conversion (Jobard et al., 2003; Heim, 2005).

It is noteworthy that the MEG effects in the present and previous experiments (Salmelin et al., 1996; Tarkiainen et al., 1999; Cornelissen et al., 2003) were essentially the same, although the tasks were different. In contrast, the fMRI stimulus effects in the LOTC seem to be sensitive to the task. Unlike in the present experiment, where a one-back task was used, a number of previous fMRI studies on reading used tasks that emphasized perceptual processing of the letter strings and found stronger LOTC activation to real words than other letter-strings (VWFA) (Cohen et al., 2002; Vinckier et al., 2007).

Figure 8 summarizes the key effects in MEG versus fMRI. In the occipital cortex, MEG and fMRI displayed similar functionality. The approximate colocalization of the MEG and fMRI activations is in line with previous studies on low-level visual processing (e.g., Sharon et al., 2007).

Marked functional differences between the MEG and fMRI measures were detected in the LOTC (MEG: letter > symbol strings, fMRI: symbol > letter strings). As discussed above, MEG evoked responses in the LOTC seem to be relatively insensitive to the task demands, whereas fMRI BOLD signals appear to be more task specific. Furthermore, modulatory effects of attention have been shown to affect the fMRI activation and also the late, but not early, MEG activation in the LOTC when processing faces and houses (Furey et al., 2006). Thus, fMRI signal from the LOTC could be dominated by late and long-lasting neural activation (Cornelissen et al., 2003) and be more susceptible to top-down modulation than the MEG evoked responses that may emphasize the bottom-up processing of written words to a higher degree. In the present one-back reading task, the participants may have attended the more demanding stimulus categories (symbols and noisy words) more carefully, resulting in an increased fMRI activation, in line with a recent EEG-fMRI comparison (Brem et al., 2009). Although there is a clear dissociation between the fMRI BOLD and MEG evoked responses, a future analysis of, e.g., MEG oscillatory activity might reveal a closer relationship to fMRI BOLD signals.

The present data successfully reproduced the typical pattern of activation in reading (Jobard et al., 2003; Salmelin, 2007), with the pronounced LIFG activation in PMRI and STG activation in MEG. The weak frontal activation detected in MEG did not differentiate words from other stimulus types, unlike the PMRI LIFG activation. In the STG, MEG responses were enhanced to words and pseudowords versus consonants. fMRI activation in the same location did not differentiate between these stimulus types, although fMRI activation was detected when contrasting letter strings to symbol strings and noisy words. The present direct MEG versus $\mathrm{AMRI}$ comparison thus verifies the differences sug- 
gested by scrutiny of previously published studies that have used only one of these imaging methods.

The present data indicates several differences between the MEG and fMRI views to the analysis of written words, when using typical analysis methods. Some spatial variance can be expected, even for the same underlying neural sources, due to possible sources of localization error in both MEG and fMRI (e.g., coregistration of data to MR images, spatial resolution of MEG, spatial smoothing in fMRI, and architecture of the local vasculature). More importantly, however, our data demonstrated marked differences in the functional roles of the MEG versus fMRI activations, although the simultaneously recorded EEG suggests that largely the same underlying neuronal responses were elicited in the two settings. Independent of the underlying reasons for the differences, these findings should be kept in mind when interpreting published studies.

Some of the observed differences may arise from the fact that MEG and fMRI probe different aspects of cerebral function (Nunez and Silberstein, 2000). MEG signals arise from the synchronous electrical activation of neurons (postsynaptic potentials of pyramidal neurons) (Hämäläinen et al., 1993), whereas the fMRI BOLD signal is a measure of blood deoxyhemoglobin that is influenced by changes in cerebral blood flow, volume, and oxygen extraction rate (Ogawa et al., 1993). Therefore, nonsynchronous electrical activation, which may well generate considerable metabolic requirements, could remain undetected with MEG. Conversely, due to the slow generation of the hemodynamic response (seconds), the fMRI signal integrates activation from a long time interval and may reflect summation of several successive activations in a given region, thus emphasizing longerlasting electrical activations while possibly downplaying the brief activations typically seen in early time windows using MEG (Furey et al., 2006). This can make fMRI sensitive to top-down, modulatory signals (as well as to bottom-up signals) and could explain the functional difference between the early MEG activation and $\mathrm{AMRI}$ activation in the LOTC observed here.

In the next step, having established that differences exist between the commonly used measures of MEG evoked responses and fMRI BOLD signal, it will be important to ask whether one can identify electromagnetic and hemodynamic markers that would yield more similar results of neural involvement. For example, MEG evoked responses in the frontal regions are typically rather weak in silent reading, but frontal regions do appear as nodes in functional connectivity analysis (Kujala et al., 2007). Furthermore, if the cortical response is not accurately time and phase locked to the stimulus, it can remain undetected in MEG/ EEG evoked responses but might show as modulation of cortical rhythmic activity. The relationship between the BOLD signal and the underlying neural events remains unresolved, but it has been suggested that changes in cortical rhythms covary with hemodynamic signal changes (e.g., Lachaux et al., 2007; Rosa et al., 2010); the evoked responses discussed in this paper would translate primarily to the alpha frequency band. Induced responses (eventrelated oscillations), not phase locked to stimulus presentation, at various frequency bands may map more directly to fMRI BOLD, especially when high-frequency electrophysiological responses are taken into account (e.g., Foucher et al., 2003). Similarly for fMRI, some other measure, such as water diffusion-weighted fMRI (Le Bihan, 2007), might reflect neural activation more reliably than the BOLD contrast.

In conclusion, the $\mathrm{MRI}$ BOLD activation and the MEG eventrelated responses of the same individuals display dissimilar functional patterns in several cortical regions outside the visual cortex in a reading task. The observed differences cannot be attributed to variation in the experimental procedures. Rather, they are likely to reflect different generation mechanisms of the MEG evoked responses and fMRI BOLD signals that should be taken into account when interpreting results obtained with these methods. Establishing that these differences are real should serve as a first step toward a principled combined use of MEG and fMRI.

\section{References}

Brem S, Halder P, Bucher K, Summers P, Martin E, Brandeis D (2009) Tuning of the visual word processing system: distinct developmental ERP and fMRI effects. Hum Brain Mapp 30:1833-1844.

Cohen L, Dehaene S, Naccache L, Lehéricy S, Dehaene-Lambertz G, Hénaff MA, Michel F (2000) The visual word form area: spatial and temporal characterization of an initial stage of reading in normal subjects and posterior split-brain patients. Brain 123:291-307.

Cohen L, Lehéricy S, Chochon F, Lemer C, Rivaud S, Dehaene S (2002) Language-specific tuning of visual cortex? Functional properties of the Visual Word Form Area. Brain 125:1054-1069.

Cohen L, Martinaud O, Lemer C, Lehéricy S, Samson Y, Obadia M, Slachevsky A, Dehaene S (2003) Visual word recognition in the left and right hemispheres: anatomical and functional correlates of peripheral alexias. Cereb Cortex 13:1313-1333.

Cornelissen P, Tarkiainen A, Helenius P, Salmelin R (2003) Cortical effects of shifting letter position in letter strings of varying length. J Cogn Neurosci 15:731-746.

Croizé AC, Ragot R, Garnero L, Ducorps A, Pélégrini-Issac M, Dauchot K, Benali H, Burnod Y (2004) Dynamics of parietofrontal networks underlying visuospatial short-term memory encoding. Neuroimage 23:787-799.

Dale AM, Sereno MI (1993) Improved localization of cortical activity by combining EEG and MEG with MRI cortical surface reconstruction: a linear approach. J Cogn Neurosci 5:162-176.

Dale AM, Liu AK, Fischl BR, Buckner RL, Belliveau JW, Lewine JD, Halgren E (2000) Dynamic statistical parametric mapping: combining fMRI and MEG for high-resolution imaging of cortical activity. Neuron 26:55-67.

Dien J (1998) Issues in the application of the average reference: review, critiques, and recommendations. Behav Res Meth Ins C 30:34-43.

Duvernoy HM (1999) The human brain, surface, blood supply, and threedimensional sectional anatomy. Vienna: Springer.

Fischl B, Sereno MI, Tootell RB, Dale AM (1999) High-resolution intersubject averaging and a coordinate system for the cortical surface. Hum Brain Mapp 8:272-284.

Foucher JR, Otzenberger H, Gounot D (2003) The BOLD response and the gamma oscillations respond differently than evoked potentials: an interleaved EEG-fMRI study. BMC Neurosci 4:22.

Furey ML, Tanskanen T, Beauchamp MS, Avikainen S, Uutela K, Hari R, Haxby JV (2006) Dissociation of face-selective cortical responses by attention. Proc Natl Acad Sci U S A 103:1065-1070.

Genovese CR, Lazar NA, Nichols T (2002) Thresholding of statistical maps in functional neuroimaging using the false discovery rate. Neuroimage 15:870-878.

Goebel R, Esposito F, Formisano E (2006) Analysis of functional image analysis contest (FIAC) data with brainvoyager QX: from single-subject to cortically aligned group general linear model analysis and self-organizing group independent component analysis. Hum Brain Mapp 27:392-401.

Hämäläinen M, Hari R, Ilmoniemi R, Knuutila J, Lounasmaa O (1993) Magnetoencephalography - theory, instrumentation, and applications to noninvasive studies of the working human brain. Rev Mod Phys 65:413-497.

Hansen PC, Kringelbach ML, Salmelin R (2010) MEG: an introduction to methods. New York: Oxford UP.

Heim S (2005) The structure and dynamics of normal language processing: insights from neuroimaging. Acta Neurobiol Exp (Warsz) 65:95-116.

Helenius P, Salmelin R, Service E, Connolly JF (1998) Distinct time courses of word and context comprehension in the left temporal cortex. Brain 121:1133-1142.

Jobard G, Crivello F, Tzourio-Mazoyer N (2003) Evaluation of the dual route theory of reading: a metanalysis of 35 neuroimaging studies. Neuroimage 20:693-712.

Korvenoja A, Huttunen J, Salli E, Pohjonen H, Martinkauppi S, Palva JM, 
Lauronen L, Virtanen J, Ilmoniemi RJ, Aronen HJ (1999) Activation of multiple cortical areas in response to somatosensory stimulation: combined magnetoencephalographic and functional magnetic resonance imaging. Hum Brain Mapp 8:13-27.

Koskinen M, Vartiainen N (2009) Removal of imaging artifacts in EEG during simultaneous EEG/fMRI recording: reconstruction of a highprecision artifact template. Neuroimage 46:160-167.

Kujala J, Pammer K, Cornelissen P, Roebroeck A, Formisano E, Salmelin R (2007) Phase coupling in a cerebro-cerebellar network at 8-13 Hz during reading. Cereb Cortex 17:1476-1485.

Lachaux JP, Fonlupt P, Kahane P, Minotti L, Hoffmann D, Bertrand O, Baciu M (2007) Relationship between task-related gamma oscillations and BOLD signal: new insights from combined fMRI and intracranial EEG. Hum Brain Mapp 28:1368-1375.

Laufs H, Daunizeau J, Carmichael DW, Kleinschmidt A (2008) Recent advances in recording electrophysiological data simultaneously with magnetic resonance imaging. Neuroimage 40:515-528.

Le Bihan D (2007) The 'wet mind': water and functional neuroimaging. Phys Med Biol 52:R57-R90.

Liljeström M, Hultén A, Parkkonen L, Salmelin R (2009) Comparing MEG and fMRI views to naming actions and objects. Hum Brain Mapp 30:1845-1856.

Lin FH, Belliveau JW, Dale AM, Hämäläinen MS (2006) Distributed current estimates using cortical orientation constraints. Hum Brain Mapp 27:1-13.

Marinkovic K, Dhond RP, Dale AM, Glessner M, Carr V, Halgren E (2003) Spatiotemporal dynamics of modality-specific and supramodal word processing. Neuron 38:487-497.

Molins A, Stufflebeam SM, Brown EN, Hämäläinen MS (2008) Quantification of the benefit from integrating MEG and EEG data in minimum 1(2)-norm estimation. Neuroimage 42:1069-1077.

Nunez PL, Silberstein RB (2000) On the relationship of synaptic activity to macroscopic measurements: does co-registration of EEG with fMRI make sense? Brain Topogr 13:79-96.

Ogawa S, Menon RS, Tank DW, Kim SG, Merkle H, Ellermann JM, Ugurbil K (1993) Functional brain mapping by blood oxygenation level-dependent contrast magnetic resonance imaging. A comparison of signal characteristics with a biophysical model. Biophys J 64:803-812.
Pylkkänen L, Llinás R, Murphy GL (2006) The representation of polysemy: MEG evidence. J Cogn Neurosci 18:97-109.

Rosa MJ, Kilner J, Blankenburg F, Josephs O, Penny W (2010) Estimating the transfer function from neuronal activity to BOLD using simultaneous EEG-fMRI. Neuroimage 49:1496-1509.

Salek-Haddadi A, Friston KJ, Lemieux L, Fish DR (2003) Studying spontaneous EEG activity with fMRI. Brain Res Brain Res Rev 43:110-133.

Salmelin R (2007) Clinical neurophysiology of language: the MEG approach. Clin Neurophysiol 118:237-254.

Salmelin R, Hari R, Lounasmaa OV, Sams M (1994) Dynamics of brain activation during picture naming. Nature 368:463-465.

Salmelin R, Service E, Kiesilä P, Uutela K, Salonen O (1996) Impaired visual word processing in dyslexia revealed with magnetoencephalography. Ann Neurol 40:157-162.

Sharon D, Hämäläinen MS, Tootell RB, Halgren E, Belliveau JW (2007) The advantage of combining MEG and EEG: comparison to fMRI in focally stimulated visual cortex. Neuroimage 36:1225-1235.

Simos PG, Basile LF, Papanicolaou AC (1997) Source localization of the N400 response in a sentence-reading paradigm using evoked magnetic fields and magnetic resonance imaging. Brain Res 762:29-39.

Tagamets MA, Novick JM, Chalmers ML, Friedman RB (2000) A parametric approach to orthographic processing in the brain: an fMRI study. J Cogn Neurosci 12:281-297.

Tarkiainen A, Helenius P, Hansen PC, Cornelissen PL, Salmelin R (1999) Dynamics of letter string perception in the human occipitotemporal cortex. Brain 122:2119-2132.

Tarkiainen A, Cornelissen PL, Salmelin R (2002) Dynamics of visual feature analysis and object-level processing in face versus letter-string perception. Brain 125:1125-1136.

Vartiainen J, Parviainen T, Salmelin R (2009a) Spatiotemporal convergence of semantic processing in reading and speech perception. J Neurosci 29:9271-9280.

Vartiainen J, Aggujaro S, Lehtonen M, Hultén A, Laine M, Salmelin R (2009b) Neural dynamics of reading morphologically complex words. Neuroimage 47:2064-2072.

Vinckier F, Dehaene S, Jobert A, Dubus JP, Sigman M, Cohen L (2007) Hierarchical coding of letter strings in the ventral stream: dissecting the inner organization of the visual word-form system. Neuron 55: 143-156. 